\title{
Computer simulations for biological aging and sexual reproduction*
}

\author{
DIETRICH STAUFFER $^{1,2 * *}$, PAULO M.C. DE OLIVEIRA ${ }^{2 * * *}$, SUZANA MOSS DE OLIVEIRA $^{2}$, \\ THADEU J.P. PENNA ${ }^{2}$ and JORGE S. SÁ MARTINS ${ }^{3}$

\footnotetext{
${ }^{1}$ Institute for Theoretical Physics, Cologne University, D-50923 Köln, Germany (Permanent address)

${ }^{3}$ Colorado Center for Chaos and Complexity / CIRES, University of Colorado, Boulder CO 80309, USA
} \\ ${ }^{2}$ Instituto de Física, Universidade Federal Fluminense, Av. Litorânea, s/n - Boa Viagem, 24210-340 Niterói, RJ
}

Manuscript received on November 22, 2000; accepted for publication on November 29, 2000.

\begin{abstract}
The sexual version of the Penna model of biological aging, simulated since 1996, is compared here with alternative forms of reproduction as well as with models not involving aging. In particular we want to check how sexual forms of life could have evolved and won over earlier asexual forms hundreds of million years ago. This computer model is based on the mutation-accumulation theory of aging, using bits-strings to represent the genome. Its population dynamics is studied by Monte Carlo methods.
\end{abstract}

Key words: parthenogenesis, genome, menopause, testosterone, Monte Carlo simulation.

\section{INTRODUCTION}

Can physicists contribute to understand biological subjects? Since the first attempts by the Nobel laureate Schrödinger (1944), there were a lot of tentative answers to this question, probably most of them useless. What particular knowledge can physicists bring to Biology? One particular tentative, biased answer for this second question is presented below. It is biased because it concerns just the authors' traditional line of research.

Critical phenomena appear in macroscopic physical systems undergoing continuous phase transitions. An example is water crossing the critical temperature of $374^{\circ} \mathrm{C}$, above which one can no longer distinguish liquid from vapour. Another is a ferromagnetic material which loses its spontaneous magnetisation when heated above its critical tem-

\footnotetext{
*Invited paper

**Foreign Member of Academia Brasileira de Ciências (ABC)

*** Member of ABC

Correspondence to: Paulo M.C. de Oliveira

E-mail: pmco@if.uff.br
}

perature. Such systems present unusual behaviours. For instance, some quantities increase without limits as one approaches more and more the critical point, as the water compressibility: a very small pressure leads to an enormous volume shrinkage. Analogously, by applying a very small magnetic field, one can drastically increase the magnetisation of a ferromagnetic sample. In both examples, also the specific heat diverges at the critical point, meaning that the system can absorb or deliver a large amount of heat, without any sensible temperature variation. Needless to mention the important practical applications of such a behaviour, according to which a fine tuning of some quantity can lead to an enormous variation of another related quantity. All modern electronics, for instance, is based on the possibility of getting an electric current passing through an otherwise insulating device, simply by applying a small electric field. Also, some plastic materials can undergo very large volume expansions under very small electric or magnetic impulses: they are used for manufac- 
turing of artificial muscles, catheters which unblock arteries, microengines, etc.

These features have attracted the attention of physicists since more than a century. They discovered an also unusual behaviour concerning the mathematical description of such systems: the appearence of power-laws, i.e. $Q \sim\left|T-T_{c}\right|^{-\gamma}$ or $C \sim\left|T-T_{c}\right|^{-\alpha}$, where $Q$ is the quoted diverging quantity (compressibility or magnetic susceptibility), $C$ is the specific heat, and $\left|T-T_{C}\right|$ measures how far the system is from its own critical point. The symbol $\sim$ represents proportionality. Critical exponents like $\gamma, \alpha$, etc are characteristic of the corresponding quantity, $Q, C$, etc.

The most interesting feature of these phenomena is the so-called universality: the precise values of the exponents $\gamma, \alpha$, etc are the same for entire classes of completely different systems. For instance, $\alpha=0.12$ for both water and any ferromagnet in which magnetisation presents uni-axial symmetry. Also, $\gamma=1.24$ for both the water compressibility and the magnetic susceptibility of the ferromagnetic material. Besides critical exponents, many other qualitative and quantitative characteristics of the various systems belonging to the same universality class coincide as well. In spite of having been observed much before, these coincidences remained unexplained until the work of Wilson (1971), three decades ago, who was awarded with the Nobel prize because of this work (see also: Wilson \& Kogut 1974, Wilson 1979). The key concept needed to understand this phenomenon is the decaying of correlations with increasing distances. Suppose one picks two points inside the system, separated by a distance $x$. How much a perturbation performed at one of these points will be felt at the other? The correlation $I$ between these two points is a measure of this mutual influence, and generally decays for larger and larger values of $x$, according to the exponential behaviour

$$
I \sim \exp (-x / \xi)
$$

(for non critical situations),

where $\xi$ is the so-called correlation length. Would one take two points distant from each other a distance $x$ larger than $\xi$, the correlation $I$ would be negligible. This means that one does not need to study the macroscopic system as a whole, with its enormous number of component units: it is enough to take a small piece of the system with linear dimensions of the same order as $\xi$ (for instance, a sphere with radius, say, $10 \xi$ ). Once one knows, for instance, the specific heat of this small piece, that of the whole system is obtained by a simple volume proportionality $C \sim V$ or $Q \sim V$.

However, the nearer the system is to its critical point, the larger is $\xi$, and the larger is the "small" piece representing the whole, i.e. $\xi \sim\left|T-T_{c}\right|^{-v}$. Just at the critical point, one can no longer break the system into small pieces: the macroscopic critical behaviour of the system is no longer proportional to its volume. Instead, critical quantities become non-linear, non-extensive, and behave as $Q \sim V^{\Phi_{\gamma}}$ or $C \sim V^{\Phi_{\alpha}}$, where $\Phi_{\gamma}=\gamma / 3 v, \Phi_{\alpha}=\alpha / 3 v$, etc. Also, the above exponential form (NC) for $I$ concerns only the dominating decay valid for a finite $\xi$. At the critical point where $\xi \rightarrow \infty$, however, other sub-dominating terms enter into the scene, i.e.

$$
I \sim x^{-\eta}
$$

(for critical situations),

where $\eta$ is another critical exponent.

Both forms (NC) and (C) mean that correlations decay for larger and larger distances. The important conceptual difference is that in (NC) they decay much faster, according to a characteristic length scale $\xi$ above which correlations become negligible. On the opposite, there is no characteristic length scale in the critical case $(\mathrm{C})$ : correlations are never negligible even between two points very far from each other, inside the system. Thus clusters and holes are observed at all sizes, a crucial property e.g. for electrophoresis.

This is a big mess for theoretical physicists: since any tentative to break such systems into small pieces is denied, they are forced to treat them as a whole. Fortunately, this same strange non-linear, critical behaviour leads to another very important 
property: most microscopic details of the system are irrelevant in what concerns its critical behaviour, since large distances dominate the scenario. That is why water compressibility presents exactly the same critical exponent $\gamma$ as the magnetic susceptibility of any uni-axial ferromagnet, as well as the same values for the other exponents $\alpha, v, \eta$, etc, and thus the same critical behaviour. Not only water and such ferromagnetic materials, but also any other natural or artificial system which belongs to the same (huge) universality class. One example of such mathematical toys is the famous Ising model: each point on a regular lattice holds a binary variable (a number 0 or 1 ), and interacts only with its neigbouring sites. No movement at all, no molecules, no atoms, no electrons interacting through complicated quantum rules. The only similarities between this toy model and real water are two very general ingredients: the three-dimensionality of the space and the one-dimensionality of the main variables involved (the numbers 0 or 1 within the toy, and the liquid-vapour density difference within water, also a number, as opposed to a three-dimensional vector). Nevertheless, one can use this very simple toy in order to obtain the critical behaviour common to all much more complicated systems belonging to the same universality class.

However, even the study of these toy models is far from trivial, due to the already quoted impossibility of breaking the system into small, separate pieces. Thus, the main instrument is the computer, where one can store the current state of each unit, i.e. a number 0 or 1 , into a single bit of the memory. By programming the computer to follow the evolution of this artificial system time after time, i.e. by repeatedly flipping 0 s into 1 s (or vice-versa) according to some prescribed microscopic rule, one can measure the various quantities of interest. Note that this approach has nothing to do with the numerical solution of a well posed mathematical problem defined by specific equations. Instead, the idea is to simulate the real dynamical behaviour of the system on the computer, and to measure the interesting quantities. During the last half century, this "almost experimental" technique was tremendously developed by the (now-called) computational physicists, a fast growing scientific community to which the authors belong (Stauffer \& Aharony 1994, de Oliveira 1991, Moss de Oliveira et al. 1999a).

Biological evolution (Darwin 1859) also presents the same fundamental mathematical ingredients which characterise physical critical systems: the power-laws. A lot of evidences are known, today (see, for instance, Kauffman 1993, 1995, Bak 1997). A simple and well known example is the number $A$ of still alive lineages within an evolving population: it decays in time according to the power-law $A \sim t^{-1}$, where the exponent -1 can be exactly obtained from the coalescence theory (see, for instance, Excoffier 1997). According to this, after many generations, all individuals of the population are descendents of a single lineage-founder ancestor. The number of generations one needs to wait for this coalescence is proportional to the number of founder individuals, due to the value -1 of the exponent. Also, during the whole evolution of the population, the number $E$ of already-extinct lineages with $n$ individuals behaves as $E \sim n^{-0.5}$, where the new exponent -0.5 is also exactly known. The interesting point is that these exponents are universal, i.e. their precise values do not change for different microscopic rules dictating how individuals die, how they are born, etc. Another simple example is the evolution of a recessive disease: the frequency of the recessive gene among the evolving population also decays in time as a power-law, thus without a characteristic extinction time. Due to this particular mathematical decaying feature, the recessive gene extinction is postponed forever (Jacquard 1978). An explanation for the narrow relation between biological evolution and critical dynamics is presented by de Oliveira (2000).

The Penna model for biological aging (Penna 1995 ) is entirely based on Darwinian evolution, and may be compared with the Ising model, for this particular evolutionary phenomenon: genes are also represented by binary variables ( 0 for ordinary genes, 1 for harmful ones). It spreads widely dur- 
ing the last half decade, and was applied to many different biological problems involving aging, always within the general interpretation above: a very simple model supposed to reproduce the universal features of much more complicated, real phenomena.

Senescence, or biological aging, can mean many things; for computer simulations it is best defined as the increase of mortality with increasing age. It seems not to exist for bacteria, where even the concept of death is difficult to define, but for humans as well as for other organisms (Vaupel et al. 1998) this rapid increase of the probability to die, after childhood diseases are overcome, is well known. Fig. 1 shows typical human data for a rich country.

The reasons for aging are controversial (Watcher \& Finch 1997, see also the whole special issues of La Recherche: July/August 1999 and Nature: November 9th, 2000). There may be exactly one gene for longevity, or senescence comes from wear and tear like for insect wings and athlete's limbs, from programmed cell death (apoptosis - Holbrook et al. 1996), from metabolic oxygen radicals destroying the DNA (see for instance Azbel 1994), or from mutation accumulation (Rose 1991). The computer simulations reviewed here use this last assumption, which does not exclude all the other reasons. For example, the oxygen radicals may produce the mutations which then accumulate in the genome transmitted from one generation to the next. Except if stated otherwise, the mutations here are all detrimental and inherited.

After a short description of the model in section 2 , we deal in section 3 with the question whether sexual reproduction was better or worse than asexual reproduction hundreds of million years ago when sex appeared, while section 4 tries to explain why today's women live longer than men and have menopause. Section 5 reviews other aspects, and Section 6 gives a short summary.

A more detailed account, but without the results of 1999 and 2000 emphasized here, is given in our book (Moss de Oliveira et al. 1999a).

\section{THE PENNA MODEL}

In the original asexual version of the Penna model (Penna 1995) the genome of each individual is represented by a computer word (bit-string) of 32 bits (each bit can be zero or one). It is assumed that each bit corresponds to one "year" in the individual lifetime, and consequently each individual can live at most for 32 "years". A bit set to one means that the individual will suffer from the effects of a deleterious inherited mutation (genetic disease) in that and all following years. As an example, an individual with a genome $10100 \ldots$ would start to become sick during its first year of life and would become worse during its third year when a new disease appears. In this way the bit-string represents in fact a "chronological genome". The biological motivation for such a representation is, for instance, the Alzheimer disease: its effects generally appear at old ages, although the corresponding defective gene is present in the genetic code since birth

The extremely short size of the 32 bit-string used in the model would be totally unrealistic if all our genes were related to life-threatening diseases. However, among the average number of $10^{8}$ units we have in our real genome, only around $10^{4}$ to $10^{5}$ units play a functional role. Moreover, only a subgroup of these will give rise to a serious disease at some moment of the individual lifetime. Besides, qualitatively there was no difference when 32, 64 and 128 bits were taken into account (Penna \& Stauffer 1996).

One step of the simulation corresponds to reading one bit of all genomes. Whenever a new bit of a given genome is read, we increase by one the individual's age. The rules for the individual to stay alive are: 1) The number of inherited diseases (bits set to 1) already accumulated until its current age must be lower than a threshold $T$, the same for the whole population. In the example given above, if $T=2$ the individual would live only for 2 years. 2) There is a competition for space and food given by the logistic Verhulst factor $V=1-N(t) / N_{\max }$, where $N_{\text {max }}$ is the maximum population size the environ- 


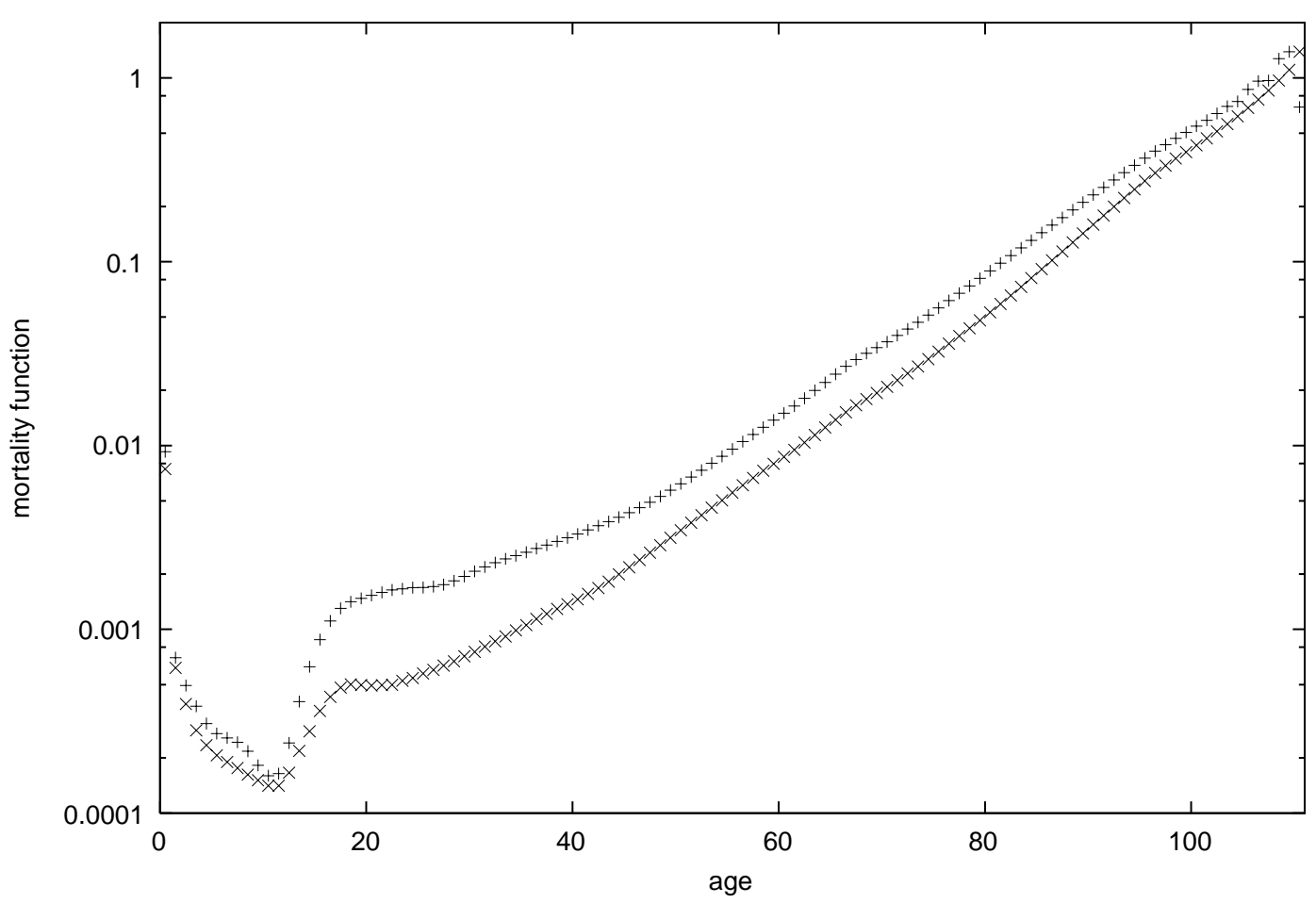

Fig. 1 - Male (+) and female (x) mortality functions in the USA, 1991-1995; from J.R. Wilmoth's Berkeley Mortality Database demog.berkeley.edu/wilmoth/. The straight line through the male data indicates the exponential increase with age (Gompertz law). These mortality functions are defined as $-d \ln S(a) / d a$ where $S(a)$ is the probability to survive up to an age of $a$ years.

ment can support and $N(t)$ is the current population size. We usually consider $N_{\max }$ ten times larger than the initial population $N(0)$. At each time step and for each individual a random number between zero and one is generated and compared with $V$ : if it is greater than $V$, the individual dies independently of its age or genome. The smaller the population size is, the greater is the probability of any individual to escape from this random killing factor.

If the individual succeeds in staying alive until a minimum reproduction age $R$, it generates $b$ offspring in that and all following years (unless we decide to set also some maximum reproduction age). The offspring genome is a copy of the parent's one, except for $M$ randomly chosen mutations introduced at birth. Although the model allows good and bad mutations, generally we consider only the bad ones.
In this case, if a bit 1 is randomly tossed in the parent's genome, it remains 1 in the offspring genome; however, if a bit zero is randomly tossed, it is set to 1 in the mutated offspring genome. In this way, for the asexual reproduction the offspring is always as good as or worse than the parent. Even so, a stable population is obtained, provided the birth rate $b$ is greater than a minimum value, which was analytically obtained by Penna and Moss de Oliveira (1995). In fact, the population is sustained by those cases where no mutation occurs, when a bit already set to 1 in the parent genome is chosen. These cases are enough to avoid mutational meltdown, that is, extinction due to accumulation of deleterious mutation, first considered by Lynch and Gabriel (1990). The reason why we consider only harmful mutations is that they are 100 times more frequent than the 
backward ones (reverse mutations deleting harmful ones - Pamilo et al. 1987).

The sexual version of the Penna model was first introduced by Bernardes (1995, 1996), followed by Stauffer et al. (1996) who adopted a slightly different strategy. We are going to describe and use the second one (see also Moss de Oliveira et al. 1996). Now individuals are diploids, with their genomes represented by two bit-strings that are read in parallel. One of the bit-strings contains the genetic information inherited from the mother, and the other from the father. In order to count the accumulated number of mutations and compare it with the threshold $T$, it is necessary to distinguish between recessive and dominant mutations. A mutation is counted if two bits set to 1 appear at the same position in both bitstrings (inherited from both parents) or if it appears in only one of the bit-strings but at a dominant position (locus). The dominant positions are randomly chosen at the beginning of the simulation and are the same for all individuals.

The population is now divided into males and females. After reaching the minimum reproduction age $R$, a female randomly chooses a male with age also equal to or greater than $R$ to breed (for sexual fidelity see Sousa \& Moss de Oliveira 1999). To construct one offspring genome first the two bit-strings of the mother are cut in a random position (crossing), producing four bit-string pieces. Two complementary pieces are chosen to form the female gamete (recombination). Finally, $m_{f}$ deleterious mutations are randomly introduced. The same process occurs with the male's genome, producing the male gamete with $m_{m}$ deleterious mutations. These two resulting bit-strings form the offspring genome. The sex of the baby is randomly chosen, with a probability of $50 \%$ for each one. This whole strategy is repeated $b$ times to produce the $b$ offspring. The Verhulst killing factor already mentioned works in the same way as in the asexual reproduction.

A very important parameter of the Penna model is the minimum reproduction age $R$. According to mutation accumulation-theory, Darwinian selection pressure tries to keep our genomes as clean as possi- ble until reproduction starts. For this reason we age: mutations that appear early in life are not transmitted and disappear from the population, while those that become active late in life when we barely reproduce can accumulate, decreasing our survival probability but without risking the perpetuation of the species. One of the most striking examples of such a mechanism is the catastrophic senescence of the pacific salmon and other species called semelparous: In these species all individuals reproduce only once in life, all at the same age. This can be easily implemented simply by setting a maximum reproduction age equal to $R$. After many generations, the inherited mutations have accumulated in such a way that as soon as reproduction occurs, individuals die. This explanation was given by Penna et al. (1995), using the Penna model (see also Penna \& Moss de Oliveira 1995 and a remark from Tuljapurkar on page 70 in Wachter \& Finch 1997).

\section{COMPARISON OF SEXUAL AND ASEXUAL REPRODUCTION}

\subsection{DeFinitions}

In this section we check which way of reproduction is best: Sexual, asexual or something in between. We denote as asexual (AS) and sexual (SX) the simulation methods described in the previous section, that means cloning of a haploid genome for AS, and crossover for diploid genomes with males and females separated for SX. Intermediate possibilities which will also be compared are apomictic parthenogenesis (AP), meiotic parthenogenesis (MP), hermaphroditism (HA), and mixtures of them. One could also group AS, AP and MP into asexual and HA and SX into sexual reproduction. Parasex, the exchange of haploid genome parts between different bacteria, is not simulated here.

To find out which way is the most successful one we simulate each choice separately with the same parameters, in particular with the same $N_{\max }$ for the Verhulst factor taking into account the limits of space and food. The choice with the largest equilibrium population, after the initial transient phe- 
nomena are overcome, is regarded as the best. We assume it would win in a Darwinian selection (see Stauffer et al. 2000 for some justification) if different populations following these different ways of reproduction would compete against each other in the same environment, without any symbiosis or predator-prey relation between them.

AS and SX were defined already in the preceding section. For AP the diploid genome is copied without crossover, only mutations. For MP the diploid genome is crossed over, and one of the two resulting haploid bit-strings is randomly chosen, duplicated and mutated to form the new diploid genome. HA is similar to SX except that there is no separation into males and females; instead all of them can generate offspring and each individual selects randomly a partner from the whole population to exchange genome as in SX. Fig. 2 summarizes the four versions schematically.

In all the copying of genome (bit-strings), point mutations are assumed to happen with the same probability per bit. Thus typically for AS with a genome of 32 bits we assume one mutation per generation for the whole genome, while for the diploid cases AP, MP, HA and SX we assume two. (We assume the same mutation rate for males and females in the Penna model simulations.) The birth rate is also assumed to be the same for all birth-giving individuals. For example, for AS, AP, MP and HA we have four offspring per suitable individual and per year, while for SX we have four offspring per suitable female and per year. Thus the birth rate averaged over males and females is only two instead of four. And we have to find out whether this loss of a factor of two in the average birthrate for SX is overcome by advantages not contained in the other ways of reproduction.

(For HA as simulated by Stauffer et al. (2000) during one iteration some individuals have already aged by one "year", while others have not yet aged. Results do not change much, see topmost data in Fig. 3 below, if now in one iteration we first let everybody age one time unit, and only afterwards partners are selected.)

\subsection{COMPARISON WITHOUT AGING}

The Redfield model (Redfield 1994) is an elegant model requiring much less computer time than the Penna model, but having no age structure. It is not a population dynamics model following the lifetime of each individual, but only simulates their probabilities to survive up to reproduction. The mortality increases exponentially with the number of mutations in the individual. For the sexual variant the number of mutations in the child is determined by a binomial distribution such that on average the child has as its own number of mutations half the number of the father, plus half the number from the mother. At birth, new mutations are added following a Poisson distribution, for both AS and SX. Because of the lack of an explicit genome, the forms AP, MP, HA between AS and SX were not simulated.

This model triggered many publications in the physics literature since it originally made SX much worse than AS: The average mortality was about 25 percent for AS, and did not change when the simulation switched to SX. But still the males were eating the food away from the females. Actually, the male mutation rate is much higher than the female one, and when that was taken into account the mortality with SX became much higher than for AS (Redfield 1994).

However, the picture changed drastically when we took into account (Stauffer et al. 1996) that most hereditary diseases are recessive (acting only when both father and mother had them in their transmitted genome) and not dominant (acting already when only one of the two inherited bit-strings has them). Then the mortality decreased by about an order of magnitude, and SX became much better than AS. The same drastic improvement was found when for SX the females selected only males with few mutations.

However, since the males do not give birth, these simulations (Stauffer et al. 1996) required the female birth rate for SX to be twice as high as the AS birth rate. Correcting this factor of two, and still assuming that only $20 \%$ of the mutations are domi- 


\begin{tabular}{|c|c|c|c|c|c|c|c|c|c|c|c|c|c|c|}
\hline \multirow[t]{8}{*}{1} & 0 & 1 & 0 & 0 & 1 & 0 & 1 & 0 & 0 & 1 & 0 & 1 & ( & 0 \\
\hline & & & & & 1 & 0 & 1 & 0 & 1 & 1 & 0 & 1 & ( & 1 \\
\hline & & & & & & & & & & & & v & & \\
\hline & & & & & & & & & & 1 & 0 & 1 & 0 & 1 \\
\hline & & & & & & & & & & 1 & 0 & 1 & 0 & 1 \\
\hline & & & & & & & $\checkmark$ & & & & & $\checkmark$ & & \\
\hline & \multirow{2}{*}{\multicolumn{4}{|c|}{$\downarrow$}} & 1 & 1 & 1 & 0 & 0 & 1 & 0 & 1 & & 1 \\
\hline & & & & & & & & & & & & & & \\
\hline 1 & 1 & 1 & 0 & 0 & 1 & 0 & 1 & 1 & 1 & 1 & 1 & 1 & ( & 1 \\
\hline
\end{tabular}

Fig. 2a-Schematic representation of the genomic changes for AS, AP, MP (from left to right).

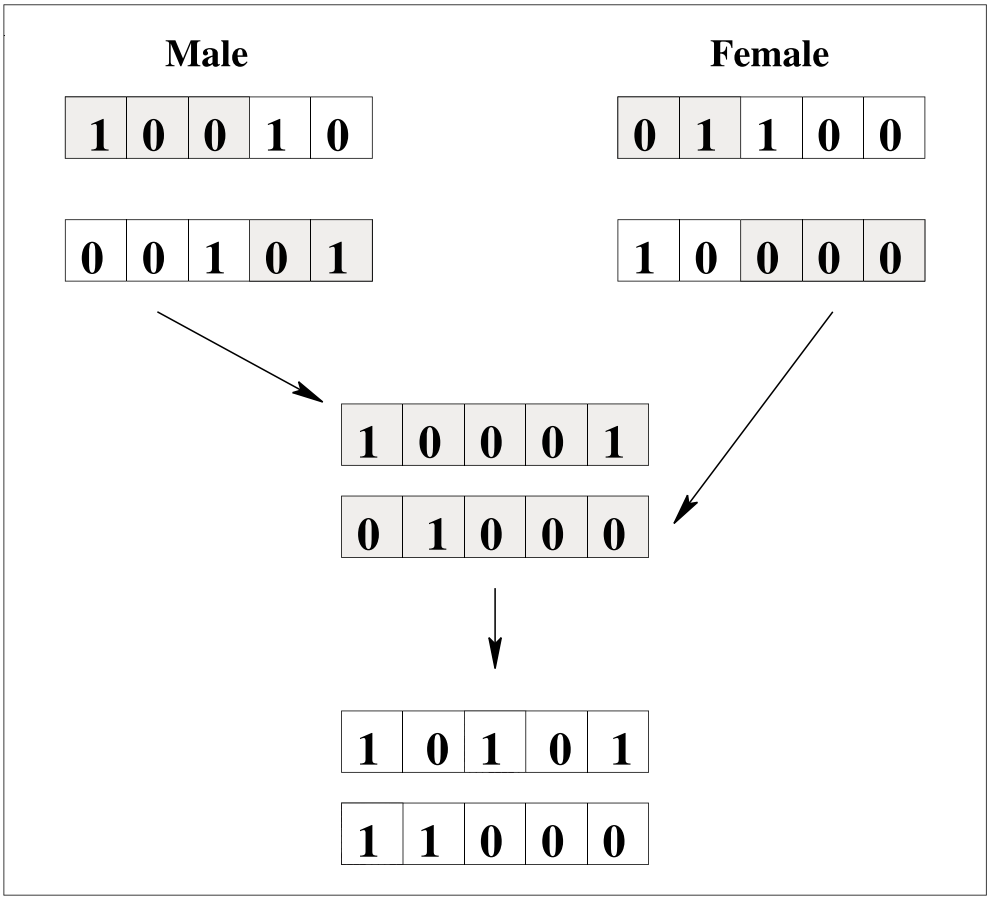

Fig. $2 b-$ Schematic representation of the genomic changes for SX. (The diagram for SX is also valid for HA, except that for HA all individuals can reproduce.) 


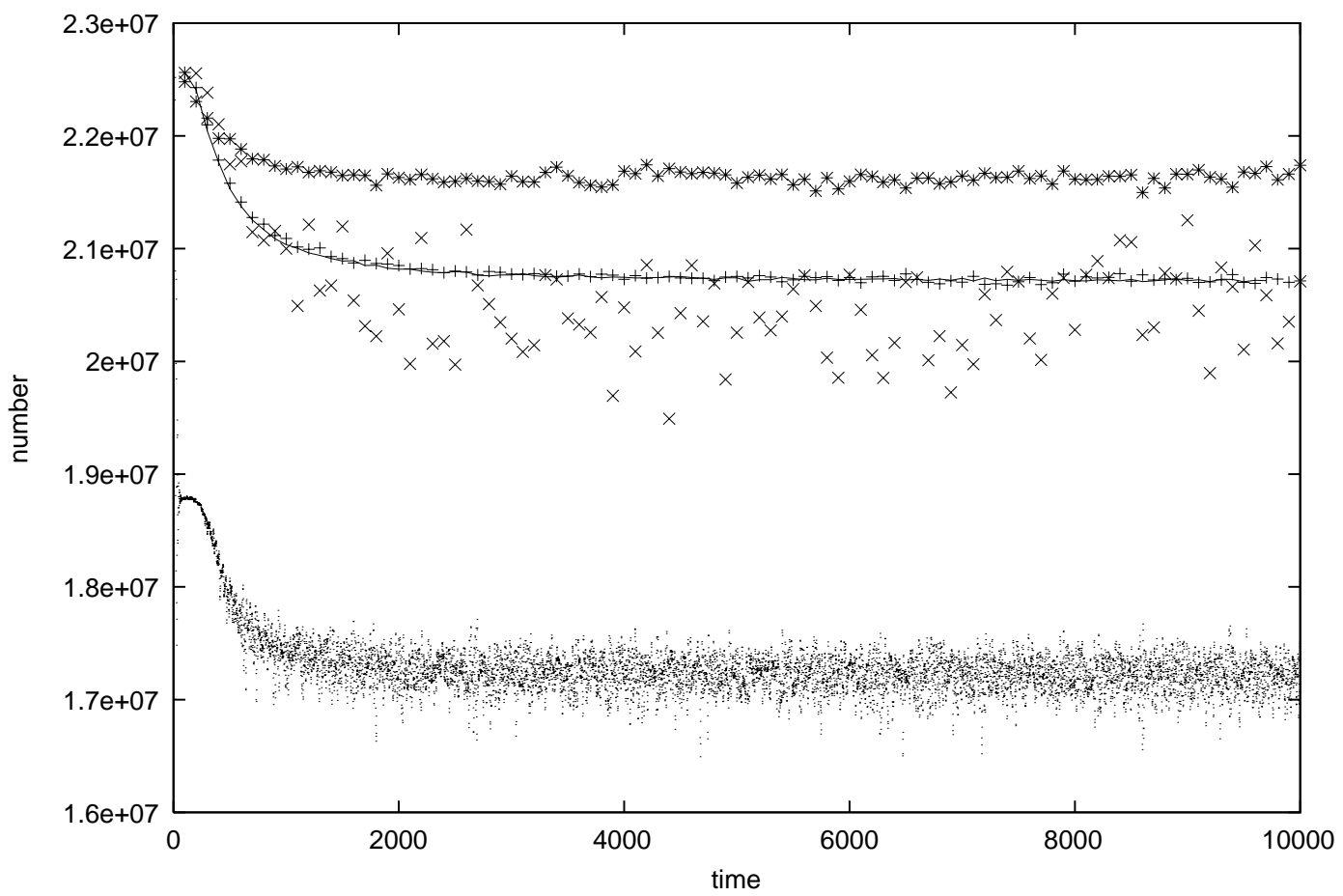

Fig. 3 - Comparison of populations, versus number of iterations or "years", for (from below) SX, AP, MP. The highest data refer to a mixture of HA and MP with $\mu=4$; nearly the same results are obtained for $\mu=3$ and 5 . The data for AS (line) overlap with those of MP (+), while AP(x) fluctuates around slightly lower values. For SX we show the sum of males and females. Threshold $T=9,4$ births per year and per female above minimum reproduction rate of 8 , one mutation per string of 32 bits at birth, $N_{\max }=80$ million is about four times larger than the actual populations.

nant diseases, SX lost out for the originally selected mutation rate (Redfield 1994) of 0.3. Increasing the mutation rate to 1 for both AS and SX, SX won (Stauffer 1999) over AS if the male mutation rate was the same as the female one, and AS won over SX when the male mutation rate was three or more times higher than the female one. Thus, as also observed in Nature, sometimes asexual and sometimes sexual reproduction is better.

A more realistic model, involving an explicit genome in the form of bit-strings, was more recently investigated by Örçal et al. (2000). It did not involve aging, however, since all bit positions were treated equally. Instead, Örçal et al. (2000) used the Jan et al. (2000) parameter $\mu$ defined such that only individuals with $\mu$ and more mutations exchange genome. (The model is then closer to HA than to SX.) Healthy individuals without many mutations reproduce similarly to AS. Five different versions were studied, depending on the number of offspring and on whether individuals with $\mu$ and more mutations mate only with each other or also with a wider population having less mutations. The simulation showed that in none of the five cases the sexual population died out; in one case it won even completely and made the AS population extinct.

These two models (Redfield 1994, Örçal et al. 2000) thus give the same result: The simpler asexual way of life is not necessarily better than the more complicated way of genome exchange. And this 
possible justification of sex comes only from intrinsic genetic reasons, not from extrinsic or social reasons like parasites, changing environment, or child protection. On the other hand, there are also cases were asexual reproduction is better. With aging included to make the simulation more realistic, the next subsection will tell us a different story.

\subsection{Comparison in Penna aging Model}

Most organisms age, and thus we should compare sexual and asexual reproduction in a model with aging, where reproduction starts only after a certain age. The Penna model of section 2 is the only one for which we know of computer simulations for aging and sex. The first comparisons of MP with SX in this model were published by Bernardes (1997). More recently, AS, AP, MP, HA and SX were simulated with it (Stauffer et al. 2000). We enlarge the range of possibilities by incorporating into it the Jan parameter (Jan et al. 2000) $\mu$ such that organisms with $\mu$ and more mutations try to find a partner with whom they exchange genome (HA and SX), while those with less than $\mu$ mutations use AP or MP. One counts only the mutations already set, up to the current age of each individual, to make the choice.

The simulated mixtures of reproduction were: MP-HA, MP-SX, AP-SX. In the first case, the final population depends non-monotonically on $\mu$ showing a maximum for intermediate $\mu$, while in the two other mixtures the behaviour is monotonic making the mixtures less interesting. Since $T$ mutations kill an individual, we have $0 \leq \mu \leq T$, with $\mu=0$ describing pure HA and $\mu=T$ describing pure MP for the MP-HA mixture. Fig. 3 summarizes our main results.

We see that SX (the small dots in the lower half) is by far the worst, MP (+) and AS (line) give nearly identical results, $\mathrm{AP}(\mathrm{x})$ is slightly worse than AS, and finally a mixture of HA and MP (stars) with $\mu=4$ gives the best results. Why then do males exist?

Some people claim men should eat less steaks and drink less alcohol. Taken to the extreme, we assume the males eat nothing and are so much smaller than the females that they consume no space. Some animals followed this way of life long before us. Then their contribution to the Verhulst dying probability $\left(N_{m}+N_{f}\right) / N_{\max }$ mentioned in section 2 becomes negligible, and the dying probability is simplified to $N_{f} / N_{\max }$. With this change, the population for SX roughly doubles, not surprisingly, and then SX is by far the best solution. The majority of the present authors insist that this result is an artifact of the assumptions and is no way to regulate their lives. In the evolution of sex hundreds of million years ago it is difficult to imagine that together with the mutation towards SX, immediately also the male body size became much smaller and consumed much less food.

Perhaps male aggressiveness plays a useful role in protecting the children while reducing the male survival chances. Using the algorithm to be described in section 4 for testosterone as an explanation for the lower mortality of women compared to men, and female partner selection described later in this section, Fig. 4 shows that now SX is above MP or AS. This child protection is already an effect outside the intrinsic genetic effects discussed before. The following paragraphs discuss environmental effects which can also justify SX over AS, in agreement with reality.

Parasites have long been claimed to justify sexual reproduction, since the greater genetic variety of the offspring gives the parasites less chance to adapt to the host. An early computer simulation (Howard \& Lively 1994) without aging already showed sexual reproduction to die out compared with asexual reproduction if no parasites are present (thus similar to Redfield 1994), while in the presence of parasites sex can give the better chance of survival (Howard \& Lively 1994). Within the more realistic Penna model, including aging, the parasite problem was studied more recently by Sá Martins (2000) with the same result: Parasites justify sex.

For this purpose, MP hosts, SX hosts and 1000 parasites were simulated together in the same environment (Sá Martins 2000) represented by $N_{\max }$ for the sum of the populations. With probability $1 / 10^{4}$, 


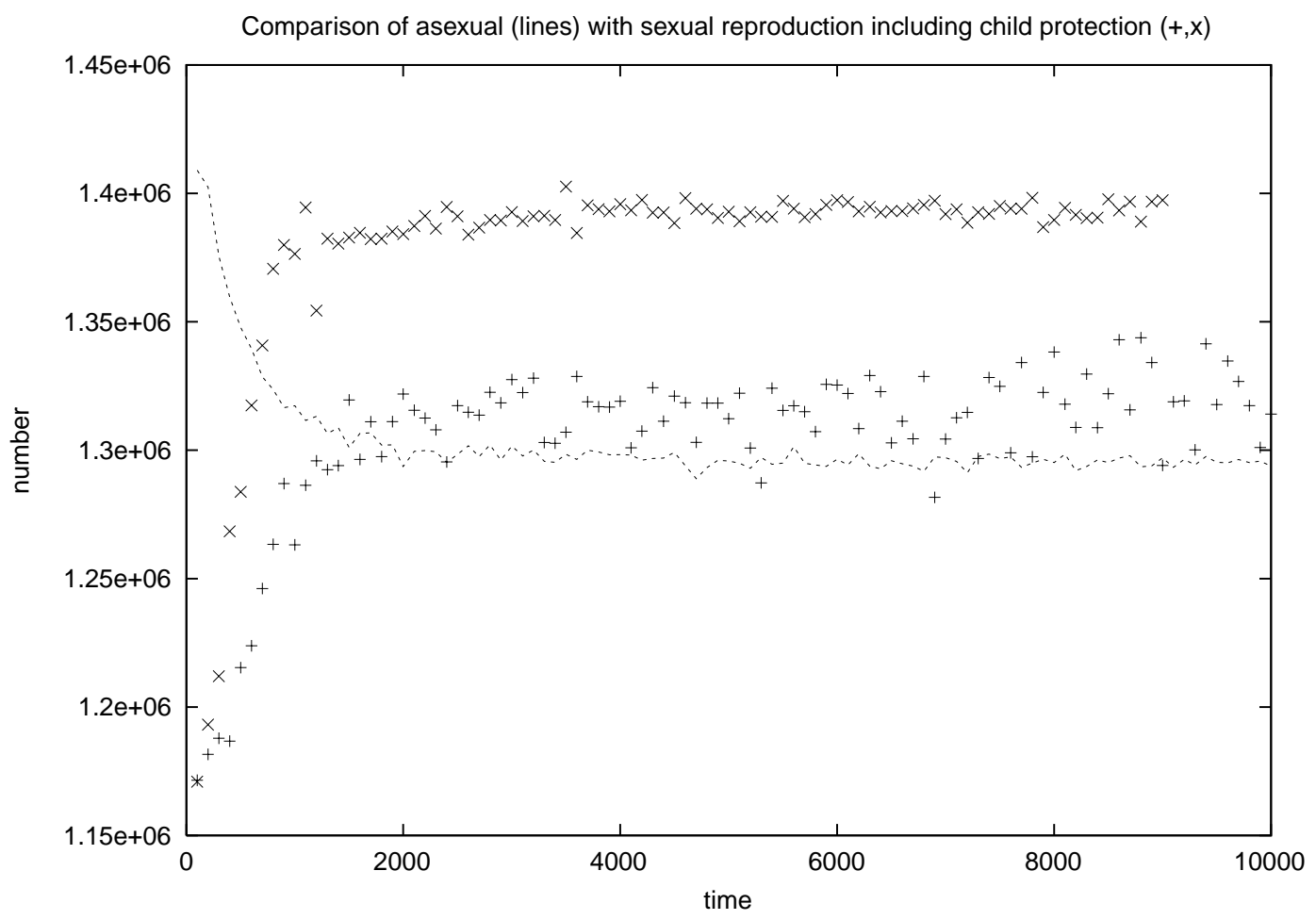

Fig. 4 - Comparison of populations, versus number of iterations, for SX with child protection $(+, \mathrm{x})$, and AS (line). (For the data marked by $\mathrm{x}$, females select only male partners with at most one active bad mutation.) $N_{\max }=5$ million; otherwise parameters as in Fig. 3.

an individual can switch from MP to SX or back. The parasites are represented by bit-strings. Each female host has contact with 60 parasites, and if one parasite agrees in its bit-string with that of the female, this host loses her ability to procreate. The parasite bit-string, on the other hand, is modified into the female bit-string if the parasite meets the same bit-string for the second time.

Starting with SX, the whole population changes to MP after a few hundred time steps, if no parasites are present. In the presence of parasites, however, starting with MP the whole population switches to SX in an even shorter time (Sá Martins 2000). Thus the well known greater variety of SX (Sá Martins \& Moss de Oliveira 1998, Dasgupta 1997) compared to MP saves the sexual population from the attacks of parasites.

Both papers (Howard \& Lively 1994, Sá Mar- tins 2000) were motivated by observations of biologist Lively and his collaborators on snails. They do not discuss if parasites already were plaguing the presumably much smaller organisms nearly $10^{9}$ years ago when sex appeared.

Another reason to justify SX compared with MP are rapid changes in the environment, to which natural evolution cannot be fast enough. SX and HA lead across the species to a larger variety in the genome than AS or MP, and after a catastrophe like the meteor killing the dinosaurs, the species with a greater variety has a larger chance to contain a minority of individuals adapted to the changed conditions. Fig. 5, adapted from Sá Martins and Moss de Oliveira (1998), compares MP with SX. First, MP gives the higher population as in Fig. 3, but when a sudden change in the environment is introduced into the simulation, the SX population has a higher 


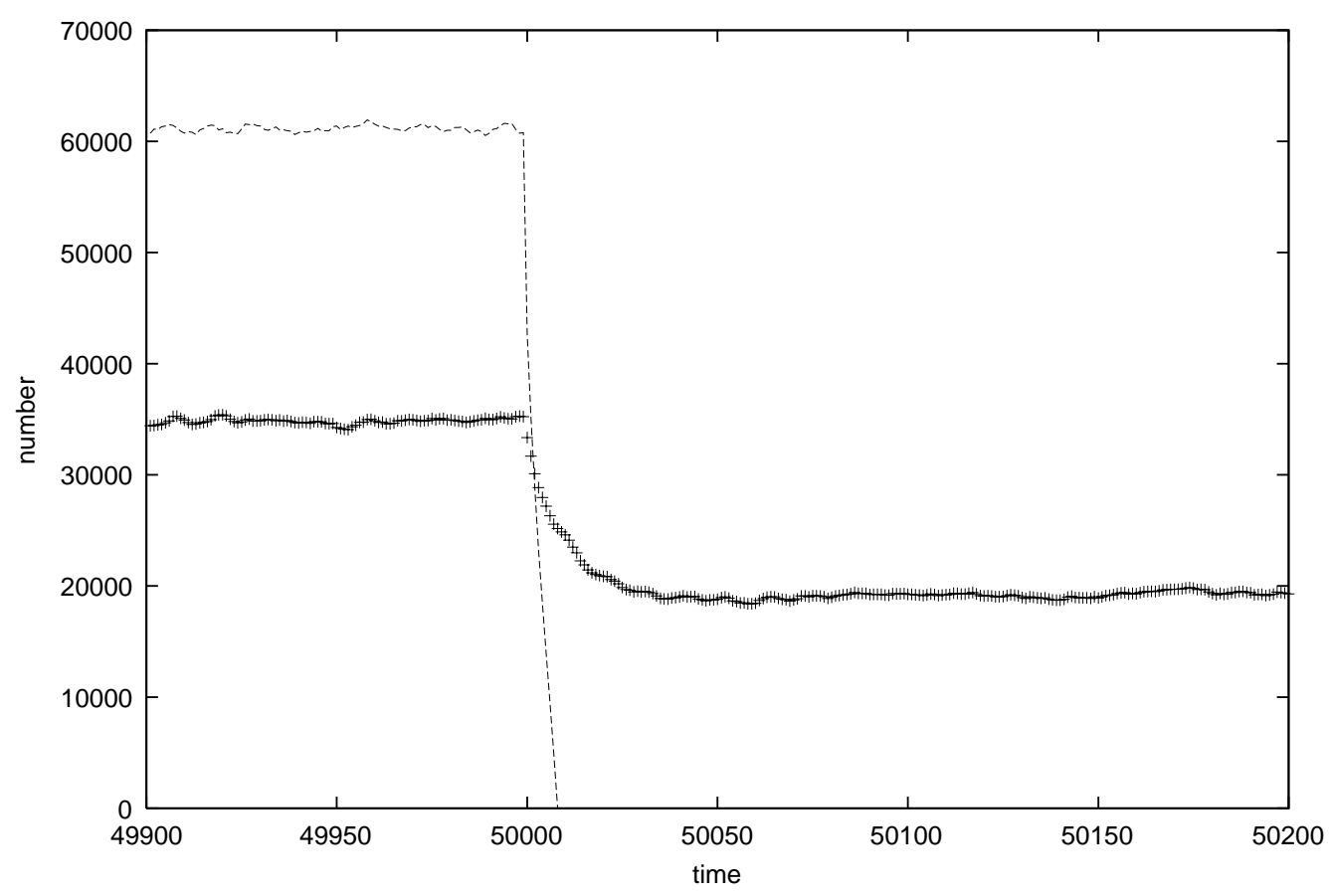

Fig. 5 - Comparison of MP (lines) with SX (crosses) population before and after a sudden change in the environment. For SX, the female birth rate of two was the same as for all MP individuals. $\left(N_{\max }=\right.$ 400000, $T=3, d=5, R=10$, one mutation per bit-string.)

chance to survive the catastrophe than MP.

Another reason why genomic exchange between different individuals may be better than AS, AP or MP is partner selection (Redfield 1994, Stauffer et al. 1996). Let us simulate in the sexual Penna model first a birthrate of eight children per female and "year"; then we reduce the birth rate to four to account for males not getting pregnant; and in the third step we assume that the females select as partners only healthy males with few mutations. Some parameter region could be found, Fig. 6, where selection in step 3 gave a strong advantage over no selection (step 2), but this advantage was not enough to overcome the loss of half the births compared with step 1. With the parameters of Fig. 3 instead, the SX data would shift upward to 18.5 million if only males with at most one active mutation are selected.

Thus, while the simpler models without aging gave clear intrinsic justifications for the existence of males, the more realistic aging model required parasites, catastrophes, or child protection for this purpose (partner selection may help somewhat) and intrinsically slightly preferred a HA-MP mixture over haploid asexual cloning. It remains to be seen what SX simulations will give in other models (e.g. Onody 2000).

\section{WHY WOMEN LIVE LONGER AND HAVE MENOPAUSE?}

Men may be useless according to section 3.3, but why do they live shorter than women, in the developed countries of the 20th century?

Many mutations limit life, and thus the higher mutation rate of males compared with females (Redfield 1994) could be the reason of higher male mortality. Simulations (Stauffer et al. 1996) showed that this is not the case: The offspring is randomly either male or female and in both cases inherits 


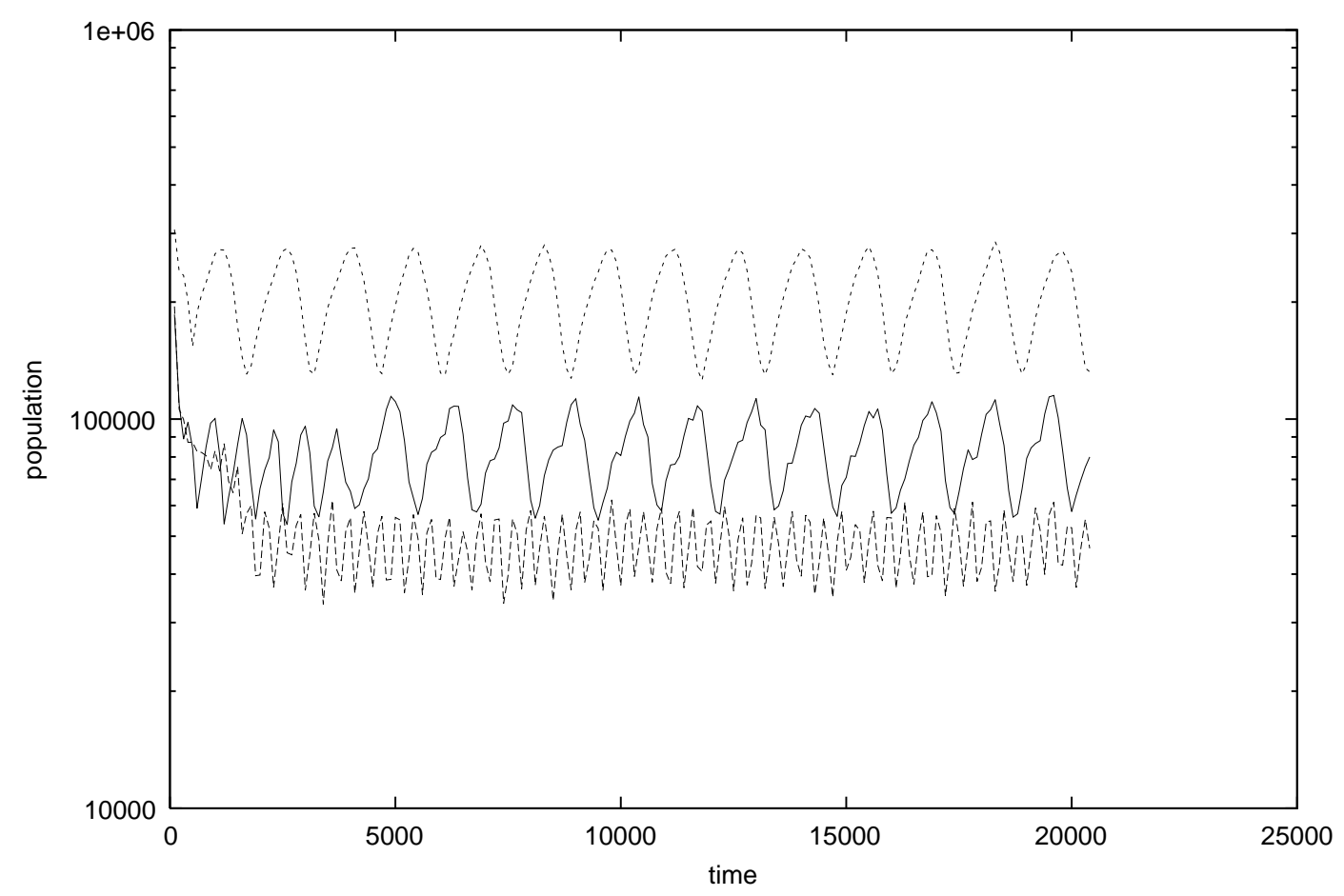

Fig. 6 - Can partner selection overcome the loss of half the births? The top data show step 1, the bottom data step 2, and the middle data step 3 (see text): Selection helps, but not enough.

roughly the same mutations from the parents; for the same reason, women are not killed by mutations after menopause (see below), in contrast to Pacific Salmon (Moss de Oliveira et al. 1999a, Penna et al. 1995). Somatic mutations, which are not inherited and not given on to the offspring, reduce the male mortality compared to the female one if the rate of somatic mutations is higher for males than for females (Moss de Oliveira et al. 1996). Alternatively, females could be just more resistant than males against diseases (Penna \& Wolf 1997). Mammalian females have two $\mathrm{X}$ chromosomes, while the males have one $\mathrm{X}$ and one $\mathrm{Y}$ chromosome such that all X mutations are dominant (Schneider et al. 1998). Except at old age, these last three assumptions all lead to higher male than female mortalities, as in reality (Fig. 1), and where reviewed in our book (Moss de Oliveira et al. 1999a).

A more recent idea was suggested to us by the medical researcher Klotz and is related to male lifestyle (steaks and alcohol) caused by testosterone (Klotz 1998, Klotz \& Hurrelmann 1998, Baulieu 1999). This hormone causes higher male aggressivity leading to death, as well as more arteriosclerosis later in life. These bad effects were perhaps counterbalanced earlier in human evolution by helping the males to defend their families against predators or fellow men. This child protection by males can in some other form also occur in other animals. Using this child protection assumption together with sexual selection as described below, the above SX results in Fig. 4 were produced. And for today's humans, the testosterone parameters of this child protection model could be chosen such that the male mortality is about twice as high as the female mortality, except at old age, in agreement with reality (Stauffer \& Klotz 2000, Stauffer 2000).

Presumably, the true reasons for the difference 
between the mortalities of men and women is a combination of genetic and social effects, as is shown by the variation from country to country within Europe (Gjonça et al. 1999). The XX-XY chromosome hypothesis (Schneider et al. 1998) is supported by the observation (Paevskii 1985) that male birds usually live longer than the females: For birds, the females have two different and the males have two identical chromosomes, opposite to mammals.

(Technical remark: To simulate the effects of male testosterone level in Fig. 4, the Verhulst dying probability was increased, for males only, by an amount proportional to the age-dependent testosterone level $k(a)$ (Stauffer \& Klotz 2000, Stauffer 2000). On the other hand, the probability of babies to survive was multiplied by a factor $\min ($ const $k(a), 2)$ such that a too low testosterone level of the father causes his babies to be killed by others. The function $k(a)$ evolved to an optimum shape through small heritable mutations in $k(a)$.)

Menopause for women means an abrupt ceasing of their reproductive function, while for men, andropause is a rather smooth decay with age. Similar effects exist for the other mammals (though perhaps under different names, which we ignore here). For Pacific Salmon, life ends for males and females shortly after the end of reproduction of both (Penna et al. 1995); why does the same effects not occur for women?

Pure genetic reasons (Stauffer et al. 1996) in the unmodified Penna model, without child care, already allow women to survive menopause. Conception decides randomly whether the new baby is a boy or a girl, and the genome is the same apart from the difference in $\mathrm{X}$ and $\mathrm{Y}$ chromosomes. Thus if all bits above reproductive age would be set equal to one for women (as for Pacific Salmon), the men would also die at that same age of menopause. To kill the women earlier than men, mother Nature would need a longevity gene in the $\mathrm{Y}$ chromosome, which in reality contains little genetic information. In this way, female survival after menopause is consistent with the mutation-accumulation hypothesis in the Penna model.
This consistency does not yet explain why menopause evolved. The only simulation we are aware of explaining menopause (Moss de Oliveira et al. 1999b) introduces two new assumptions into the Penna model: a risk of dying at birth for the mother which increases with the number of active mutations and thus with age; and child care in the sense that young children die if their mother dies. Then the maximum age for reproduction was allowed to emerge from the simulation, instead of being put in fixed at the beginning, by assuming it to be hereditary apart from small mutations up or down. As a result, the distribution of the maximum age of reproduction peaked at about 15 "years" whereas without child care its maximum was at 32 years, at the oldest possible bit position.

\section{OTHER ASPECTS}

Geneticist S. Cebrat (priv. comm.) has criticized our crossover method for the sexual Penna model as published in Moss de Oliveira et al. (1999a). Since we split the bit strings at some randomly selected position and then combine the first part of one bitstring with the second part of the other bit-string, and since bit positions correspond to individual age, we produce correlations for the mutations in consecutive ages. In real DNA, the genes are not stored consecutively in the order in which they become active during life. Thus it is better to select randomly one subset of bits from one bit-string, and the other bits from the complimentary bit-string. Simulations indicate no clear difference, Fig. 7.

Overfishing (Moss de Oliveira et al. 1995, Penna et al. 2000) and the inheritance of longevity (de Oliveira et al. 1998) were simulated using the asexual version of the Penna model.

Although we have centered this work on the Penna model, it is not the only way to go in aging studies. It is an apropriate tool that allows us to unravel the importance of mutation accumulation effects. The very first models studied by physicists, summarized in Stauffer (1994), were based on the antagonistic pleiotropy. They were inspired by the 


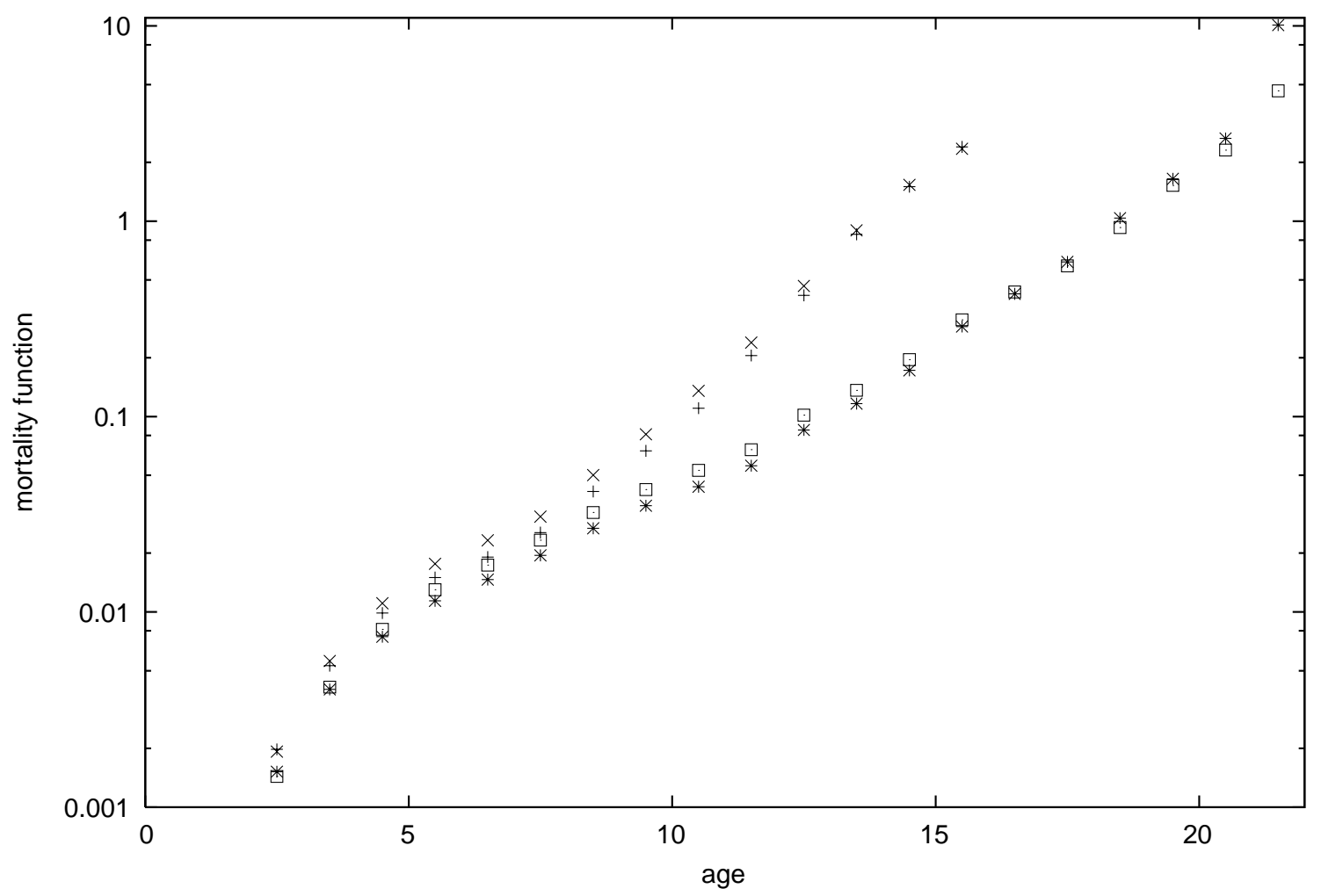

Fig. 7 - Comparison of traditional ordered crossover (x, squares) with better random crossover (+, stars) showing little difference. The Verhulst deaths appear at all ages $(+, \mathrm{x})$ or only at birth (stars, squares).

Partridge and Barton review (Partridge \& Barton 1993). There they proposed a constraint for the survival rates from babies to juveniles $J$ and juveniles to adults $A$ as $J+A^{4}=1$. Because it has only two parameters and three ages, the exponential increase of mortalities cannot be observed. Some attempts of implementing antagonistic effects on bit-string models have been done. Bernardes imposed an extra deleterious mutation at advanced ages for a fraction of the population with higher reproduction rates (Bernardes 1996). Sousa and Moss de Oliveira, in a more detailed study, have shown that the combined action of mutation accumulation and antagonistic pleiotropy at defined ages can extend the lifespan of a population (in preparation). Sousa and Penna have introduced a different strategy, where both sides of the antagonism are present: good (bad) mutations at earlier (later) ages (in preparation). The minimum age at reproduction is allowed to vary. The later the individual reaches the sexual maturity, the more fertile it is. There is a clear compromise to postpone the maturity (and consequently to decrease the integrated fertility) against to be more exposed to death by competition or action of bad mutations. Preliminary results suggest that except for unrealistic handicaps on the fertility for later maturity, natural selection drives the populaton to earliest maturity (see also Medeiros et al. 2000).

\section{CONCLUSIONS AND PERSPECTIVES}

The computer simulations of biological aging, mostly using the Penna model, could explain nicely the roughly exponential increase of mortality functions with age, the existence of menopause, and (with less clarity) the existence of other forms of reproduction besides asexual cloning of hap- 
loid genomes. We speculate that from this asexual way, mother Nature may have evolved via apomictic and/or meiotic parthenogenesis towards hermaphroditism, and only later separated the population into males and females because of external or social reasons, as simulated. Finally, menopause appeared because of the need for child care by the mother and the risks for her associated with giving birth later in life. For practical applications, simulations suggested not to catch young fish, or young and old lobsters, in order to maximize the catch (Moss de Oliveira et al. 1995, Penna et al. 2000).

\section{ACKNOWLEDGMENT}

We are indebted to the Brazilian agencies CAPES, $\mathrm{CNPq}$ and FAPERJ for partial financial support.

\section{RESUMO}

A versão sexual do modelo de envelhecimento biológico de Penna, simulada desde 1996, é comparada aqui com formas alternativas de reprodução bem como com modelos que não envolvem envelhecimento. Em particular, queremos verificar como formas sexuais de vida poderiam ter evoluído e predominado sobre formas assexuais há centenas de milhões de anos. Este modelo computacional baseia-se na teoria do envelhecimento por acumulação de mutações, usando 'bits-strings' para representar o genoma. Sua dinâmica de populações é estudada por métodos de Monte Carlo.

Palavras-chave: partenogênese, genoma, menopausa, testosterona, simulação Monte Carlo.

\section{REFERENCES}

Azbel MYa. 1994. Universal biological scaling and mortality. Proc NatlAcad Sci USA 91: 12453-12457.

BAK P. 1997. How Nature Works: the Science of SelfOrganized Criticality, Oxford University Press.

BAULIEU EE. 1999. Le vieillissement est-il soluble dans les hormones? La Recherche 322: 72-74.

BernARdes AT. 1995. Mutational meltdown in large sexual populations. J Physique I 5: 1501-1515.

BERNARDES AT. 1996. Strategies for reproduction and aging. Ann Physik 5: 539-550.
Bernardes AT. 1997. Can males contribute to the genetic improvement of the species? J Stat Phys 86: 431439.

DARWIN C. 1859. On the Origin of Species by Means of Natural Selection, Murray, London.

Dasgupta S. 1997. Genetic crossover vs. cloning by computer simulation. Int J Mod Phys C 8: 605-608.

De Oliveira PMC. 1991. Computing Boolean Statistical Models, World Scientific, Singapore/ London/ New York.

DE Oliveira PMC. 2000. Why do evolutionary systems stick to the edge of chaos. Theory in Biosci: in press.

de Oliveira PMC, Moss de Oliveira SM, Bernardes AT \& STAUfFER D. 1998. Lancet 352: 911-912.

Excoffier L. 1997. Ce que nous dit la genealogie des genes. La Recherche 302: 82-84.

Guonça A, Tomassini C \& Vaupel JW. 1999. Pourqoi les femmes survivent aux hommes? La Recherche 322: 96-99.

Holbrook NJ, Martin GR \& Lockshin RA. 1996. Cellular Aging and Death, Wiley-Liss, New York.

Howard RS \& Lively CM. 1994. Parasitism, mutation accumulation and the maintenance of sex. Nature 367: 554-557 and 368: 358 (Erratum).

JACQUARD A. 1978. Éloge de la Différence: la Génétique et les Hommes, Éditions du Seuil, Paris.

Jan N, Moseley L \& Stauffer D. 2000. A hypothesis for the evolution of sex. Theory in Biosci 119: 166168.

Kauffman SA. 1993. Origins of Order: Self-Organization and Selection in Evolution, Oxford University Press, New York.

Kauffman SA. 1995. At home in the Universe, Oxford University Press, New York.

KLotz T. 1998. Der frühe Tod des starken Geschlechts, Cuvillier, Göttingen.

Klotz T \& Hurrelmann K. 1998. Adapting the health care system to the needs of the aging male. The Aging Male 1: 20-27. 
Lynch M \& Gabriel W. 1990. Mutation load and the survival of small populations. Evolution 44: 17251737.

Medeiros G, Idiart MA \& de Almeida RMC. 2000. Selection experiments in the Penna model for biological aging. Int J Mod Phys C 11: No. 7.

Moss de Oliveira S, Penna TJP \& Stauffer D. 1995. Simulating the vanishing of northern cod fish. Physica A 215: 298-304.

Moss de Oliveira S, De Oliveira PMC \& Stauffer D. 1996. Aging with sexual and asexual reproduction: Monte Carlo simulations of mutation accumulation. Braz J Phys 26: 626-630.

Moss de Oliveira S, de Oliveira PMC \& Stauffer D. 1999a. Evolution, Money, War and Computers, Teubner, Leipzig.

Moss de Oliveira S, Bernardes AT \& Sá Martins JS. 1999b. Self-organisation of female menopause in populations with child care and reproductive risk. Eur Phys J B 7: 501-504.

ONODY RN. 2000. The Heumann-Hötzel Model revisited. Talk O-24 at FACS 2000, Maceió, Brazil.

Örçal B, Tüzel E, Sevim V, Jan N \& Erzan A. 2000. Testing a hypothesis for the evolution of sex. Int $J$ Mod Phys C 11: 973-986; and also DeCoste C \& Jan $\mathrm{N}$, priv. comm.

Paevski VA. 1985. Demography of Birds (in Russian), Nauka, Moscow.

Pamilo P, Nei M \& Li WH. 1987. Accumulation of mutations in sexual and asexual populations. Genet Res, Camb 49: 135-146.

Partridge L \& Barton NH. 1993. Optimality, mutation and the evolution of aging. Nature 362: 305-311.

PENNA TJP. 1995. A bit-string model for biological aging. J Stat Phys 78: 1629-1633.

Penna TJP \& Moss de Oliveira S. 1995. Exact results of the bit-string model for catastrophic senescence. J Physique I 5: 1697-1703.

Penna TJP \& StaufFer D. 1996. Bit-string aging model and German population. Zeits Phys B 101: 469-470.
Penna TJP \& Wolf D. 1997. Computer simulation of the difference between male and female death rates. Theory in Biosc 116: 118-124.

Penna TJP, Moss de Oliveira S \& Stauffer D. 1995. Mutation accumulation and the catastrophic senescence of the Pacific salmon. Phys Rev E 52: R3309R3312.

Penna TJP, Racco A \& Sousa AO. 2000. Can microscopic models for age-structured populations contribute to Ecology? Talk IT-11 at FACS 2000, Maceió, Brazil (to appear in Physica A).

ReDFIELD RJ. 1994. Male mutations and the cost of sex for males. Nature 369: 145-147.

Rose MR. 1991. Evolutionary Biology of Aging, Oxford University Press, New York.

SÁ Martins JS. 2000. Simulated coevolution in a mutating ecology. Phys Rev E 61: 2212-2215.

Sá Martins JS \& Moss de Oliveira S. 1998. Why sex - Monte Carlo simulations of survival after catastrophes. Int J Mod Phys C 9: 421-432.

Schneider J, Cebrat S \& Stauffer D. 1998. Why do women live longer than men? A Monte Carlo Simulation of Penna-type models with $\mathrm{X}$ and $\mathrm{Y}$ cromossomes. Int J Mod Phys C 9: 721-725.

SCHRÖDINGER E. 1944. What is Life?, Cambridge University Press, Cambridge.

Sousa AO \& Moss de Oliveira S. 1999. High reproduction rate versus sexual fidelity. Eur Phys $J$ B 10: 781-785.

STAuffer D. 1994. Monte Carlo simulations of biological aging. Braz J Phys 24: 900-906.

Stauffer D. 1999. Why care about sex? Some Monte Carlo justification. Physica A 273: 132-139.

StAufFer D. 2000. Self-organisation of testosterone level in the Penna-Klotz aging model. Theory in Biosciences, in press.

Stauffer D \& Aharony A. 1994. Introduction to Percolation Theory, Taylor and Francis, London.

Stauffer D \& Klotz T. 2000. The mathematical point of view: The sex-specific life expectancy and the in- 
fluence of testosterone in an aging simulation model and its consequences for prevention. Submitted to The Aging Male.

Stauffer D, de Oliveira PMC, Moss de Oliveira S \& ZoRzenon dos Santos RM. 1996. Monte Carlo simulations of sexual reproduction. Physica A 231: 504-514.

Stauffer D, Sá Martins JS \& Moss de Oliveira S. 2000. On the uselessness of men - Comparison of sexual and asexual reproduction. Int J Mod Phys $\mathrm{C}$ 11: No. 7.

Vaupel JW, Carey JR, Christensen K, Johnson TE, Yashin AI, Holm NV, Iachine IA, Kanisto V, Khazaeli AA, Liedo P, Longo VD, Zeng Y, Manton KG \& Curtsinger JW. 1998. Biodemography of longevity. Science 280: 855-860.
WACHTER KW \& Finch CE. 1997. Between Zeus and the Salmon. The Biodemography of Longevity, National Academy Press, Washington DC.

WILSON KG. 1971. Renormalization group and critical phenomena I. Renormalization group and the Kadanoff scaling picture. Phys Rev B4: 3174-3183.

WiLson KG. 1979. Problems in physics with many scales of length. Sci Am 241: 140-157.

WiLson KG \& Kogut J. 1974. The renormalization group and the $\epsilon$ expansion. Phys Rep 12C: 75-200. 